\title{
The early phases of a solar prominence eruption and associated flare: a multi-wavelength analysis
}

\author{
C. Chifor ${ }^{1}$, H. E. Mason ${ }^{1}$, D. Tripathi ${ }^{1}$, H. Isobe ${ }^{1,2}$, and A. Asai ${ }^{3}$ \\ ${ }^{1}$ Department of Applied Mathematics and Theoretical Physics, Centre for Mathematical Sciences, Wilberforce Road, \\ Cambridge CB3 OWA, UK \\ e-mail: [c.chifor;h.e.mason;d.tripathi]@damtp.cam.ac.uk \\ 2 Department of Earth and Planetary Science, University of Tokyo, Hongo, Bunkyo-ku, Tokyo 113-0033, Japan \\ e-mail: isobe@eps.s.u-tokyo.ac.jp \\ 3 Nobeyama Solar Radio Observatory, National Astronomical Observatory of Japan, Minamimaki, Minamisaku, Nagano, 384-1305, \\ Japan \\ e-mail: asai@nro.nao.ac.jp
}

Received 24 May 2006 / Accepted 4 August 2006

\section{ABSTRACT}

\begin{abstract}
Aims. We aim to examine the precursor phases and early evolution of a prominence eruption associated with a M4-class flare and a partial halo coronal mass ejection (CME) observed on 2005 July 27. Our main goal is to investigate the precursor eruption signatures observed in EUV, X-ray and microwave emission and their relation to the prominence destabilisation.

Methods. We perform a multi-wavelength study of the prominence morphology and motion using high-cadence and spatial resolution EUV $171 \AA$ images from the TRACE satellite. The high-temperature flare radiative emission in soft and hard X-rays are analysed through imaging and spectral modeling with RHESSI. Complementary microwave images (17 GHz and $34 \mathrm{GHz}$ ) from NoRH are also investigated.

Results. The activation of the filament proceeds from one anchored footpoint. We observe "pre-eruption" brightenings in X-ray and EUV images, close to the erupting footpoint of the prominence, being temporally correlated to the point when the prominence first enters a slow-rise phase, and then an accelerated fast-rise phase. The brightness temperature $\left(T_{\mathrm{b}}\right)$ of the prominence at $34 \mathrm{GHz}$ is increasing during the eruption. We also find very good correlation between the prominence height-time profile and the spatially integrated soft X-ray (SXR) emission.

Conclusions. We discuss the observed precursor brightenings with respect to possible mechanisms that might be responsible for the prominence destabilisation and acceleration. Our observations suggest that reconnection events localised beneath the erupting footpoint may eventually destabilise the entire prominence, causing the eruption.
\end{abstract}

Key words. Sun: prominences - Sun: coronal mass ejections (CMEs) - Sun: flares - Sun: UV radiation - Sun: radio radiation Sun: X-rays, gamma rays

\section{Introduction}

It is widely accepted that the basic process(es) responsible for the occurrence of filament eruptions, CMEs and flares are closely related and of magnetic origin (Shibata 1999; Priest \& Forbes 2002; Svestka 2001). Priest \& Forbes (2002) classify all these phenomena under the same category of "eruptive flares". Filament eruptions are one aspect of such explosive events. Filaments (referred to as prominences if observed over the solar limb) consist of cool material suspended in the corona by a core magnetic field. During eruptions, both the filament and the filament-carrying field move together (Rust 1976). When erupting filaments are associated with a CME, they are often recognised as a bright core in white light images, following a leading edge and a dark cavity (Illing \& Hundhausen 1985).

Several studies (Sterling \& Moore 2004a,b; Gretchnev et al. 2006) have unveiled a common pattern of filament eruptions: an initial "slow-rise phase" (with very small acceleration), during which the filament gradually ascends, followed rather abruptly by a transition to a "fast-rise phase" of strong acceleration. The eruption "onset" has been defined as the transition between these two phases. It should be pointed out, however, that there are two types of "onsets" to consider (which may well be closely related): one responsible for the start of the slow-rise, the other for the transition to the fast-rise.

Although it is generally accepted that filament eruptions are driven by the magnetic pressure unleashed during the explosion (Moore \& Sterling 2005; Moore 1998), it is not yet understood what causes the magnetic pressure to decrease. The question of the trigger $(s)$ initiating such eruptions remains open. In order to gain insight into their origins, it is paramount to closely scrutinise the very start of these events.

Based on a statistical analysis between the erupting filaments and the photospheric magnetic field, Feynman \& Martin (1995) found that the filament eruptions were highly correlated with an emerging magnetic bipole and its orientation. However, by a similar study, Wang \& Sheeley (1999) showed that filaments can erupt without emerging bipole, concluding that new flux might not represent a critical condition for filament destablisation. Tripathi (2005) and references therein, found that there can be different types of changes in the photospheric magnetic field - magnetic flux cancelation, new bipole emergence, evolution in a nearby pre-existing bipole - which can lead to filament 
eruption. Therefore, signatures of the destabilisation triggers do not seem to converge.

In this respect, multi-wavelength observations may prove useful for investigating eruption triggers by providing unique information to map their spatial and temporal locations. Several authors (Moore et al. 2001; Sterling et al. 2001; Sterling \& Moore 2003; Moon et al. 2004; Sterling \& Moore 2005) have reported precursor brightenings in previous studies of filament eruptions. In this paper we present a multi-wavelength analysis of a well-observed prominence eruption associated with an M3.7-class flare and CME which occurred on 2005 July 27. A preliminary study of this event has been given by Chifor et al. (2006). The location and spectral coverage of the eruption render it ideal for investigating its early phases and early evolution. The prominence erupts such that its southern connected end begins to rise. We observe pre-eruption brightenings close to the rising footpoint of the prominence in X-ray images from the Reuven Ramaty High Energy Solar Spectroscopic Imager (RHESSI) and extreme ultraviolet (EUV) images taken by TRACE. The prominence also becomes increasingly brighter in microwave images from the Nobeyama Radioheliograph (NoRH). We make a detailed study of the observed brightenings investigating their relation to possible eruption triggers.

In Sect. 2 we describe the observing instruments and multiwavelength analysis for our event. Section 3 summarises the results, while a discussion and our conclusions are included in Sect. 4.

\section{Multi-wavelength observations and data analysis}

\subsection{Overview of the event and observing instruments}

A prominence eruption occurred just over the eastern solar limb (09N 89E) between 04:00 and 05:00 UT on 2005 July 27. Approximately $5 \mathrm{~h}$ prior to eruption, the prominence can first be seen emerging from behind the limb in images taken by the EIT (Extreme-ultraviolet Imaging Telescope; Delaboudinière et al. 1995) aboard the Solar and Heliospheric Observatory(SoHO) in the $195 \AA$ (Fe XII) filter. Figure 1 displays the running difference images taken by EIT at $195 \AA$ before (top panel; 04:3604:24 UT), during (middle panel; 04:48-04:36 UT) and after (bottom panel; 05:12-04:48 UT) the eruption. EP marks the erupting prominence in the middle panel of Fig. 1. The erupting prominence was associated with a white-light CME detected by $\mathrm{SoHO} / \mathrm{LASCO} / \mathrm{C} 2$. The CME was first reported at 04:54 UT with a linear speed of $\sim 1787 \mathrm{~km} \mathrm{~s}^{-1}$ according to the LASCO CME catalogue ${ }^{1}$. Figure 2 shows the associated CME. The core of the CME is marked in the middle panel (CORE) and may be identified as the erupting prominence.

The event was mainly analysed using high-cadence and high spatial resolution TRACE EUV images taken in the $171 \AA$ filter. TRACE observes the Sun in difference EUV channels including one white-light channel. The capabilities of the TRACE instrument are described by Handy et al. (1999). Fe IX and Fe X lines dominate the emission in $171 \AA$ TRACE images, making them most sensitive to temperatures of 1-2 MK. Phillips et al. (2005) have demonstrated that the $171 \AA$ filter also has a hightemperature response due to continuum and Fe XX contributions. Thus, high-temperature ( $\sim 10-20 \mathrm{MK})$ features, usually prominent in images taken in the TRACE $195 \AA$ (Fe XXIV) band and in RHESSI X-ray images, can sometimes also be visible in the

\footnotetext{
${ }^{1}$ http://lasco-www.nrl.navy.mil/cmelist.html
}
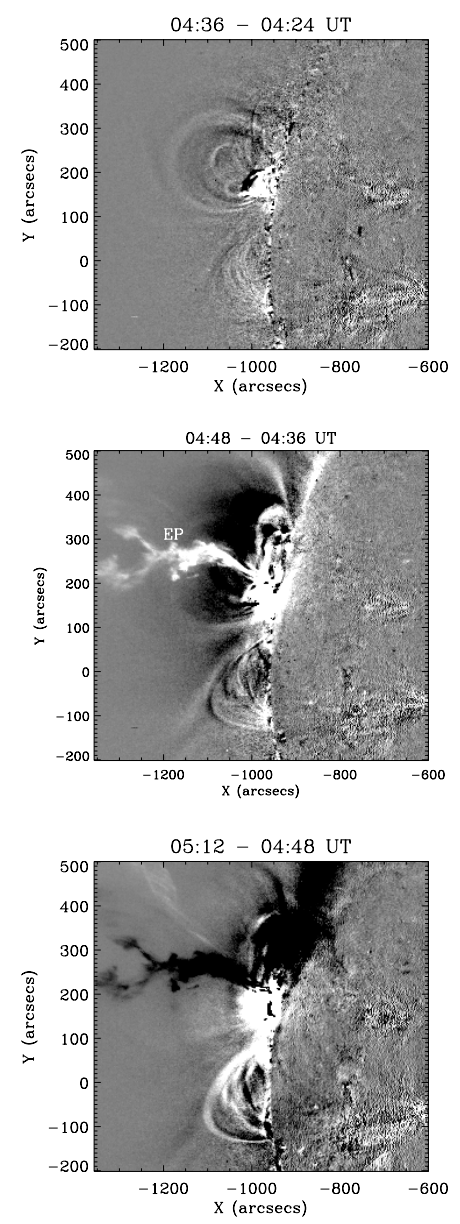

Fig. 1. Erupting prominence on 2005 July 27 observed in a running difference sequence of EIT $195 \AA$ images. Images are taken before (top panel), during (middle panel) and after (bottom panel) the eruption. EP marks the erupting prominence in the middle panel.
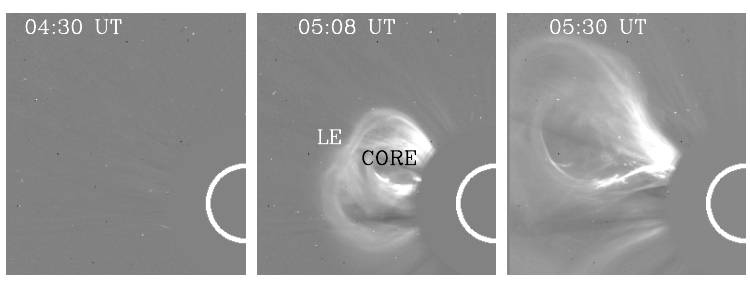

Fig. 2. Partial halo CME associated with the erupting prominence on 2005 July 27 observed in SoHO/LASCO/C2 images. The CME was first reported at 04:54 UT with a speed of $\sim 1960 \mathrm{~km} \mathrm{~s}^{-1}$ (speeds are calculated based on running difference images taken by $\mathrm{LASCO} / \mathrm{C} 2$ and C3 observations). The core (CORE) of the CME, which may be identified as the erupting prominence, as well as the leading edge (LE) are marked.

TRACE 171 images. A detailed study of the eruption observed in TRACE images (Fig. 3) is reported in Sect. 2.2.

A GOES class M3.7 flare accompanied the prominence eruption with the soft X-ray flux intensity attaining its maximum at 05:01 UT. We used RHESSI satellite (Lin et al. 2002) data to detect high-energy photons emitted during the event. Time profiles of the soft and hard X-ray (HXR) emission are shown in Fig. 4. A small pre-eruption X-ray emission enhancement can be observed in both GOES and RHESSI light-curves (around 03:5004:00 UT). Equipped with high-resolution germanium detectors, RHESSI enables a detailed analysis of flare spectra at high 

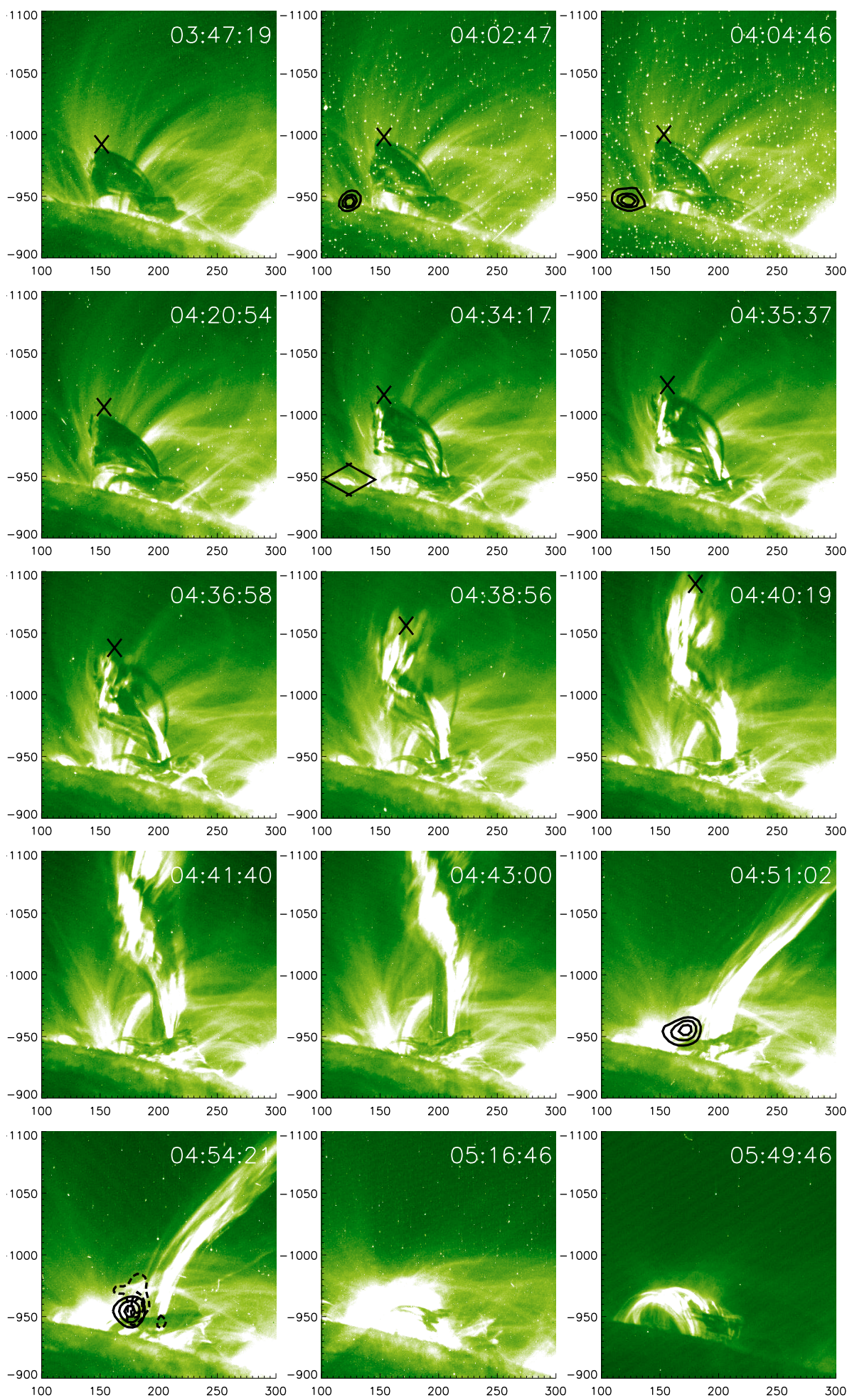

Fig. 3. TRACE $171 \AA$ A images showing the 2005 July 27 prominence eruption. Images have been rotated $90^{\circ}$ clockwise such that the heliospheric north direction is to the right. Each image is labeled with the universal time (UT) at which it was taken (upper left corner). Superimposed are simultaneous RHESSI 3-12 keV (solid curves), and 25-50 keV (dashed curves) contours (12-25 keV image contours were also taken around 04:50 UT, during the impulsive flare phase of the flare, however they roughly coincide with $3-12 \mathrm{keV}$ contours, and thus were not represented). Contour levels are shown at $60 \%, 80 \%$ and $90 \%$ of the peak intensity. The EUV brightening is marked (diamond) on the image taken at 04:34:17 UT. The prominence motion was examined by tracking the "highest" part of the prominence marked with cross symbols.

energies ( $3 \mathrm{keV}-17 \mathrm{MeV})$. The dynamic range in flare energies detectable with RHESSI is made possible by a set of attenuators, or shutters (thin aluminum disks) which can move in front of the detectors to prevent detector saturation. RHESSI spectral modeling enables measurements of the physical parameters (temperature and emission measure) describing the hottest part of thermal plasma. Furthermore, non-thermal plasma emission can be characterised by fitting models such as bremsstrahlung 

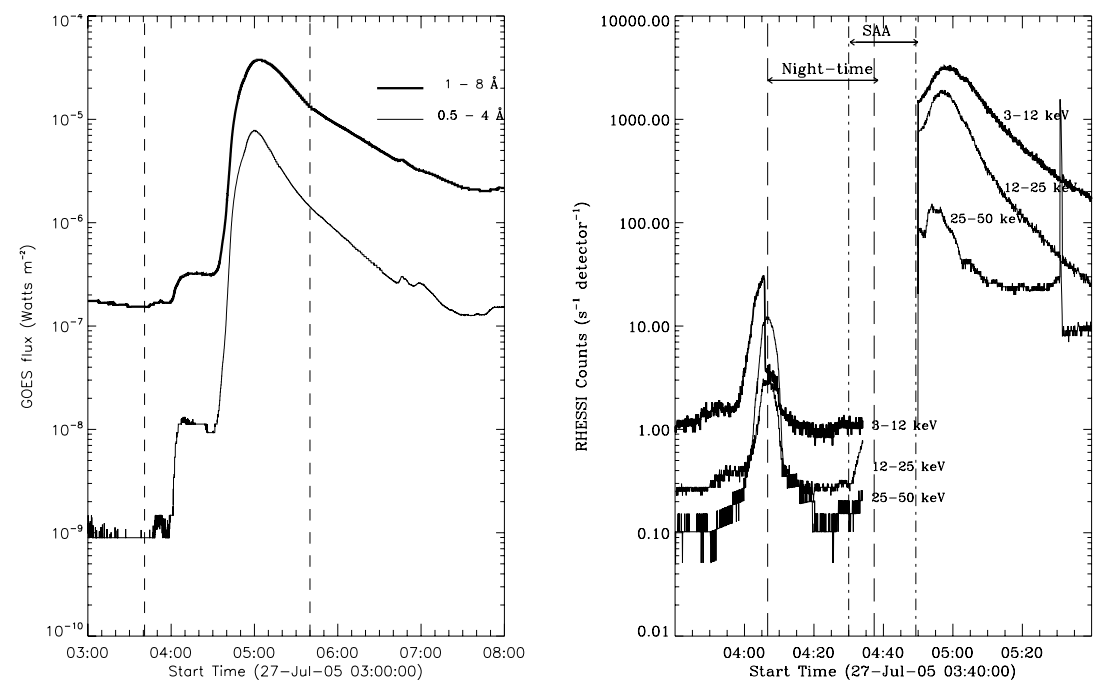

Fig. 4. Temporal evolution in X-rays. Left: SXR fluxes in the GOES $0.5-4 \AA$ and $1-8 \AA$ channels. The two dashed vertical lines mark the start and end time of RHESSI light-curves shown in the plot on the right. Right: RHESSI count rates in the energy ranges of 3-12 keV, 12-25 keV, and 25-50 keV averaged over all nine RHESSI detectors. Dashed lines indicate the period of night-time, while the time when the instrument entered the South Atlantic Anomaly is marked by dot-dashed vertical lines. Prior to entering night-time, both RHESSI shutters are out. Immediately after the data gap, the thin RHESSI attenuator comes into place. The spike observed at 05:30 UT is due to a brief removal of the thin shutter.

from a power-law mean electron distribution. RHESSI also has full-Sun imaging capabilities using rotating grids to modulate the X-ray fluxes, providing a spatial resolution as low as $\sim 2^{\prime \prime}$ (Hurford et al. 2002). Section 2.4 presents the analysis of the eruption in X-rays.

The study is further complemented by NoRH (Nakajima et al. 1994) microwave images obtained at two frequencies, $17 \mathrm{GHz}$ and $34 \mathrm{GHz}$. Microwave observations of the event are given in Sect. 2.5.

Table 1 summarises the basic characteristics of the observing instruments, along with the spatial resolution, imaging cadence and temporal coverage for our event observations. With the exception of the RHESSI satellite which went behind Earth and then entered Van Allen radiation belts during the eruption, all observing instruments provided full temporal coverage of the prominence eruption.

\subsection{Analysis of the prominence evolution in EUV TRACE images}

Figure 3 describes the motion of the prominence as it appears in TRACE 171 images which have been rotated $90^{\circ}$ clockwise such that north direction is to the right. The prominence has a complex shape, with a southernmost observed end connected at the limb. However, considering projection effects, it is possible that the prominence is curved, having one connected end hidden behind the limb, while also being connected at the observed southernmost location through a barb. Prior to 03:55-04:00 UT no significant motion is detected. After this time, we observe a very gradual but noticeable rise of the southern part of the prominence. The slow-rise can be best observed between $\sim 03: 58$ and 04:32 UT with a steady outward (away from the solar limb) motion of the prominence, rising at a constant speed of $\sim 4.8 \mathrm{~km} \mathrm{~s}^{-1}$. The rising speed was calculated by measuring the distance between the "highest" part of the prominence (marked by cross symbols in Fig. 3) and Sun centre.

At $\sim 04: 28$ UT, we observe a brightening emerging further south of the prominence footpoint (marked with a diamond in Fig. 3 at 04:34:17 UT). Between 04:29 and 04:32 UT the sudden heating of a prominence magnetic thread was observed at the southern connected part of the prominence (Fig. 5), with an EUV brightening of this strand shooting upwards (away from the limb). These brightenings appeared shortly before the transition to an accelerated phase of the prominence at 04:32 UT. During this quick evolution of the fast-rise phase, we observe more strands becoming bright in EUV, threading the entire prominence.

Finally, cooling loop arcades are forming just beyond the limb, with a residual filament/prominence observed in front of them. We observe these final stages of evolution until approximately 06:00 UT.

It is known that because TRACE is not a full disk imager, its absolute pointing is incorrect (see e.g. Gallagher et al. 2002). Therefore, care must be taken in comparisons between TRACE observations and other instruments such as RHESSI or NoRH. EUV images taken by EIT come to the rescue in this respect, since the instrument provides full disk observations. In order to correct for TRACE's pointing, we cross-correlated the TRACE images with nearly-simultaneous EIT 195 images, following the method described by Gallagher et al. (2002). As a result, TRACE images were shifted by $\sim 2.5^{\prime \prime}$ in the solar $x$ direction and $\sim-22^{\prime \prime}$ in the solar $y$ direction. Because the calculated offsets are significant (especially in the $y$ direction), care was taken to ensure the validity of the result. Several cross-correlations were made at different times during the event, and the shift was found to be the same for all comparisons. Figure 6 shows one example of EIT intensity contours plotted over a TRACE image after correction.

\subsection{Height-time analysis of the erupting prominence seen by TRACE and corresponding GOES $X$-ray fluxes}

The correlation between the motion of the erupting prominence and the X-ray emission was studied. Figure 7 represents the height-time profile (stars) for the erupting prominence. The gradual, slow-rise may be observed prior to 04:32 UT. We calculated a speed of $4.8 \mathrm{~km} \mathrm{~s}^{-1}$ from the slope of a least squares linear fit (dashed line). After 04:32 UT, the height vs. time curve was 
Table 1. Multi-wavelength instrumental coverage of the 2005 July 27 event.

\begin{tabular}{|c|c|c|c|c|c|}
\hline Instrument & Spectral coverage & Wavelength bands & $\begin{array}{l}\text { Spatial resolution } \\
(\operatorname{arcsec})\end{array}$ & Cadence & $\begin{array}{c}\text { Temporal coverage } \\
\text { (UT) }\end{array}$ \\
\hline RHESSI & X-ray & $3 \mathrm{keV}-17 \mathrm{MeV}$ & $\sim 8$ & $20-120 \mathrm{~s}$ & $\begin{array}{c}03: 01-04: 06 \text { and } \\
04: 50-05: 41\end{array}$ \\
\hline GOES & X-ray & $\begin{array}{c}0.5-4 \AA \text { and } \\
1-8 \AA\end{array}$ & - & - & 03:00-06:00* \\
\hline TRACE & EUV & $171 \AA$ & 0.5 & $40 \mathrm{~s}$ & 03:00-06:00* \\
\hline EIT & EUV & $195 \AA$ & 2.6 & $12 \mathrm{~min}$ & 00:00-06:00* \\
\hline NoRH & Microwave & 17 and $34 \mathrm{GHz}$ & $\begin{array}{c}14^{\prime \prime}(17 \mathrm{GHz}) \text { and } \\
7^{\prime \prime}(34 \mathrm{GHz})\end{array}$ & $1 \mathrm{~s}$ & 22:45 (on 2005 Jul. 26)-06:30* \\
\hline
\end{tabular}

* Full coverage of the event. Start and end times were chosen to span an ample temporal range during which the eruption occurs: from the very appearance of the prominence over the limb until it has "fully" erupted.

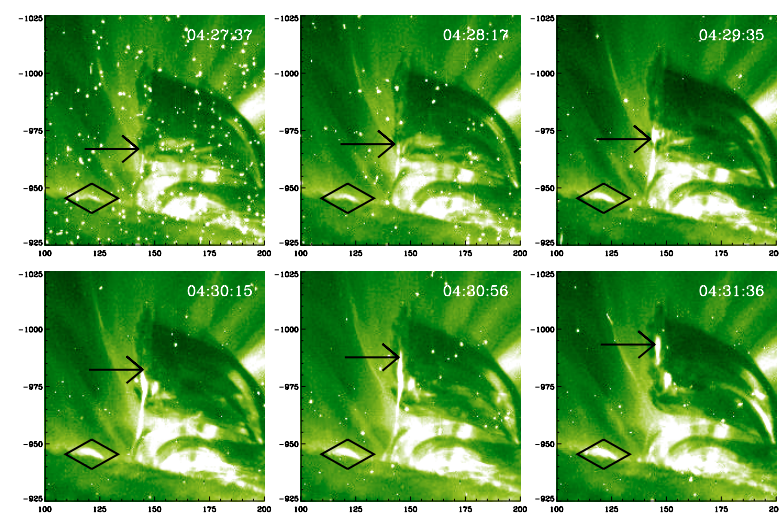

Fig. 5. Fast heating of a prominence magnetic thread, rapidly moving upwards, at the prominence footpoint (indicated by right arrows), during the slow-rise phase of the eruption. The movement is suggestive: the brightened magnetic strand appears to be "cut off" after heating. The EUV brightening feature south of the footpoint is marked by diamonds. These events occur slightly before the transition to the fast-rise phase of the eruption, which was observed at 04:32 UT. Images are marked with the corresponding universal time (UT) (upper left corner).

modeled with a second order polynomial, from which the velocity and acceleration were determined. During this phase, the prominence speed was found to increase almost linearly, with a constant acceleration of about $0.48 \mathrm{~km} \mathrm{~s}^{-2}$, reaching a maximum of $300 \mathrm{~km} \mathrm{~s}^{-1}$ at 04:41 UT. After this time, the rising part of the prominence goes beyond the TRACE field-of-view (FOV), reaching its maximum displacement from the solar centre (about $850000 \mathrm{~km}$ ). In Fig. 7 we overplotted the GOES SXR lightcurves in the 1-8 $\AA$ band (thick curve) showing a clear correlation with the height-time profile of the prominence.

\subsection{Pre-eruption brightenings and flare evolution in $X$-ray emission: RHESSI imaging and spectral analysis}

An enhancement in the SXR emission was observed in both GOES and RHESSI lightcurves between approximately 03:50 and 04:01 UT. After 04:01 UT, the increasing count rate enabled us to obtain RHESSI images in the $3-12 \mathrm{keV}$ energy band. Figure 3 shows 3-12 keV RHESSI image contours overlaid on simultaneous TRACE images (at 04:02:47 and 04:04:46 UT). The increase in X-ray emission was observed near-simultaneous with the slow-rise onset. The X-ray contours were also found spatially coincident with the EUV brightening observed approximately $30 \mathrm{~min}$ later in TRACE images. After 04:04 UT, the RHESSI spacecraft enters night-time, followed by the South

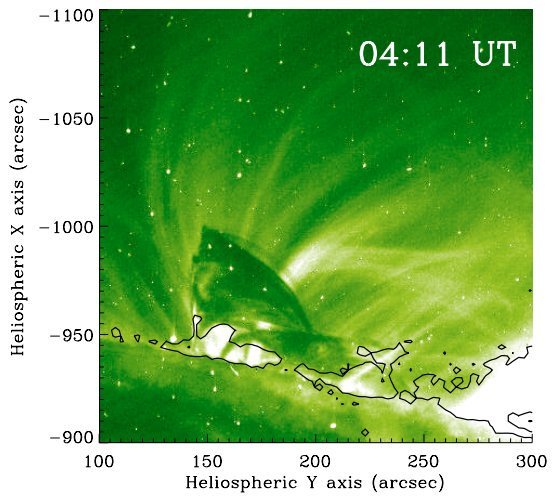

Fig. 6. EIT $195 \AA$ contours superimposed on TRACE $171 \AA$ image after correcting for TRACE's pointing following the method outlined in Gallagher et al. (2002). The TRACE and EIT images are nearly simultaneous at 04:11 UT. TRACE images were shifted by $\sim 2.5^{\prime \prime}$ in the solar $x$ direction and $\sim-22^{\prime \prime}$ in the solar $y$ direction. Both contours and corrected image have been rotated $90^{\circ}$ clockwise.

Atlantic Anomaly (SAA), making it impossible to track the soft $\mathrm{X}$-ray images during this period (Fig. 4). Later X-ray images were taken in $3-12,12-25$ and $25-50 \mathrm{keV}$ bands, during the next RHESSI orbit, when the flare associated with the eruption reaches its impulsive stages. Figure 3 (images at 04:51:02 and 05:54:21 UT) shows the X-ray emission during the impulsive phase of the flare located at a different site, $\sim 50^{\prime \prime}$ shifted north of the earlier precursor brightenings.

In order to obtain RHESSI images and spectra, one must account for instrumental effects such as pileup. Strong pileup is more likely to occur at high count rates, during RHESSI observations with no attenuators in front of the detectors. Prior to entering night-time, the shutters are out. However, during the precursor flare phases, the countrate is not very high (less than 40 counts $\mathrm{s}^{-1}$ detector $^{-1}$ ) while a pileup check assured a livetime of $\sim 97 \%$ which should ensure safe observations. Furthermore, particle precipitation events were reported just prior to nighttime, and thus we were required to carefully avoid these effects.

We applied the Clean algorithm available in the standard RHESSI software to reconstruct RHESSI images from grids 3, $4,5,6,8$ and 9 using an integration time of 20 to $120 \mathrm{~s}$. The combination of detectors, time range and energy bins was carefully chosen in order to ensure good spatial and temporal resolution while satisfying the requirement that enough counts are used to make a reliable image (according to Hurford et al. 2002, at least $\sim 10^{3}$ photons are required).

RHESSI spectral analysis during the precursor emission was also performed. Spectra were obtained using $1 / 3 \mathrm{keV}$ energy 


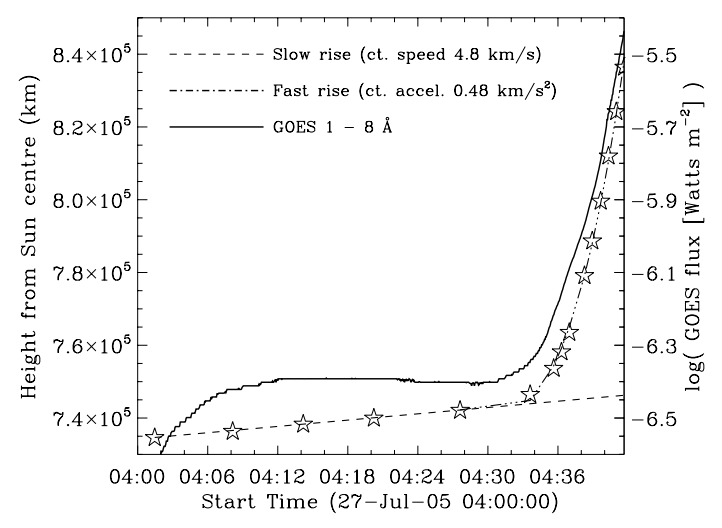

Fig. 7. Erupting filament motion as observed from TRACE 171 images. Heights of the uppermost point of the filament (represented by stars) were calculated from Sun's centre. The time profile of the filament height was fitted with a linear function between 04:02 and 04:32 UT (dashed line). From the slope of the linear fit, we calculated a speed of $4.8 \mathrm{~km} \mathrm{~s}^{-1}$ during the slow-rise phase of the eruption. Between 04:32 and 04:41 UT, the height vs. time profile was fitted with a secondorder polynomial (dashed-dot-dot curve). A constant acceleration of $0.48 \mathrm{~km} \mathrm{~s}^{-2}$ was calculated for the fast-rise eruption phase. During this phase, the velocity increases linearly up to $300 \mathrm{~km} \mathrm{~s}^{-1}$. GOES soft Xrays in the 1-8 $\AA$ channels are overplotted (thick curve), showing good correlation with the height-time profile.

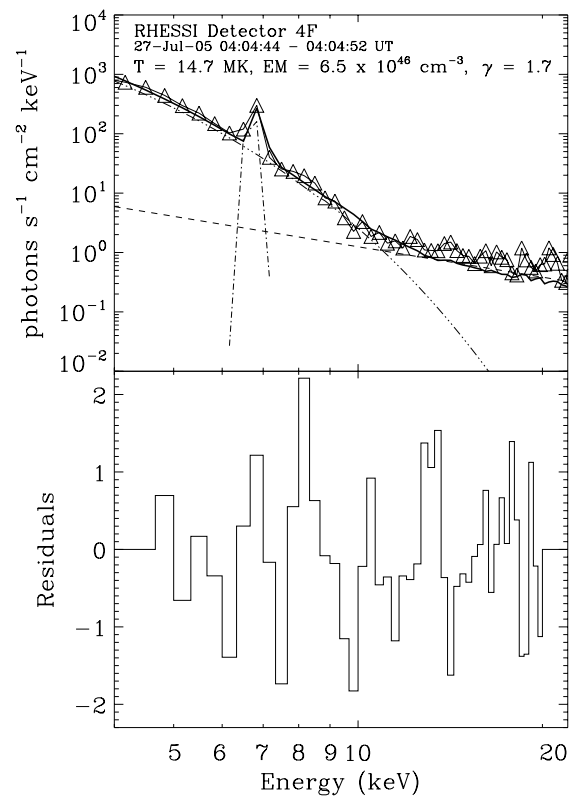

Fig. 8. Upper panel: RHESSI spectrum (triangles) accumulated between 04:04:44 and 04:04:52 UT, using detector 4. The thermal spectrum was modeled with one isothermal component (dashed-dot-dot curve) and one Gaussian function fitting the Fe line feature (dashed-dot curve). The high-energy part of the spectrum was fitted with a broken power law bremsstrahlung component (dashed curve) with low-energy cutoff. The sum of all these models, the best-fit model, is represented by the thick, continuous curve. The fitting range used was 4-20 keV. Lower panel: fit residuals representing the number of standard deviations of the best-fit model above or below data.

bins at energies less than $25 \mathrm{keV}$ and $12 \mathrm{~s}$ time intervals. Spectral fitting was achieved using the Object Spectral Executive (OSPEX) which has recently incorporated the CHIANTI (Landi et al. 2006) atomic code into its software. Prior to fitting, background was subtracted from night-time intervals, avoiding the particle precipitation events. The thermal plasma emission was modeled with an isothermal component (describing the free-free and free-bound continuum) and one Gaussian function to fit the $6.7 \mathrm{keV}$ Fe line feature (method described in detail by Phillips et al. 2006). The temperature and emission measure parameters were derived from the slope of the thermal continuum model. The observed high-energy emission $(\sim 10 \mathrm{keV})$ was fitted with a non-thermal component which has a power-law distribution $I(\varepsilon)$ $\propto \varepsilon^{-\gamma}$, where $\gamma$ represents the power-law index of the spectrum. An example of a fitted RHESSI spectrum using detector 4 between 04:04:44 and 04:05:52 UT is given in Fig. 8. The fitting shows that, at energies above $10 \mathrm{keV}$, the non-thermal contribution is predominant over the thermal component.

The thermal energy of the RHESSI source may be estimated using the temperature and emission measure derived from spectral modeling and the following expression:

$$
E_{\text {thermal }}=3 \cdot k_{\mathrm{B}} \cdot T \cdot \sqrt{E M \cdot V \cdot q}
$$

where $k_{\mathrm{B}}$ is the Boltzmann constant, $T$, the temperature, $E M$ is the emission measure, $V$, the source volume and $q$ represents the filling factor assumed to be equal to unity for a homogeneous medium. At the time of the brightening, $E M$ is approximately $10^{46} \mathrm{~cm}^{-3}$ and $T$ is about $15 \mathrm{MK}$. The volume was determined from RHESSI images, assuming a spherical source. From these parameters, the thermal energy was estimated to be of the order $\sim 10^{29}$ ergs.

Moreover, we note that hard X-ray bursts (above $25 \mathrm{keV}$ ) detected with RHESSI occur well past the start of the fast-rise phase (at about 04:55 UT), as shown in Fig. 4.

\subsection{Analysis of the prominence evolution in microwave $\mathrm{NoRH}$ images}

The prominence eruption was also observed in NoRH microwave images obtained at two frequencies: $17 \mathrm{GHz}$ and $34 \mathrm{GHz}$. The dominant emission mechanism of quiescent prominences in microwaves is thermal bremsstrahlung. The free-free emission is often considered to be optically-thick at $17 \mathrm{GHz}$ (Rao \& Kundu 1977; Gretchnev et al. 2006). In the opticallythick case, $T_{\mathrm{b}}$ is assumed to be equal to the kinetic temperature. In the present case, $T_{\mathrm{b}}$ measured at $17 \mathrm{GHz}$ is about $8000 \mathrm{~K}$ before the eruption, comparable to $T_{\mathrm{b}}$ measured at $34 \mathrm{GHz}$. The small difference between the $T_{\mathrm{b}}$ of the prominence at $17 \mathrm{GHz}$ and that at $34 \mathrm{GHz}$ is probably due to the presence of the optically-thin coronal plasma. Nevertheless, we can assume that the emission of the observed prominence is almost opticallythick, even at $34 \mathrm{GHz}$.

The left panels of Fig. 9 show the TRACE and NoRH images taken at 04:02 UT. The shape of the prominence observed in NoRH microwave images is roughly the same as that in TRACE EUV images. In the right panel of Fig. 9 we present the time profile of the $34 \mathrm{GHz} T_{\mathrm{b}}$ averaged over the selected region represented in the left panels of Fig. 9. We can see that $T_{\mathrm{b}}$ starts to increase in both frequencies at 03:50 UT, approximately at the slow-rise onset. However, it is difficult to determine exactly the start time of the increase due to the noise level of about $1000 \mathrm{~K}$. In particular, the $34 \mathrm{GHz}$ images include artificial modulation patterns due to the side lobe.

On the other hand, we often observe non-thermal emissions associated with strong energy release processes at these frequencies. The emission mechanism is mainly gyrosynchrotron radiation. Although we have found evidence of non-thermal emission in RHESSI X-ray observations (as discussed in Sect. 2.4), 

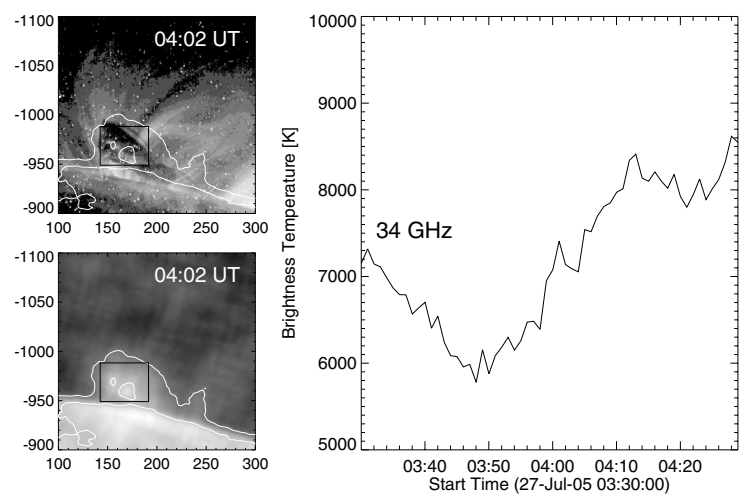

Fig. 9. Upper left: A NoRH $34 \mathrm{GHz}$ contour superimposed on a TRACE 171 image taken at 04:02 UT (corrected for TRACE pointing). Both the NoRH contour and the TRACE image have been rotated $90^{\circ}$ clockwise. The rectangular region used for calculating the brightness temperature $\left(T_{\mathrm{b}}\right)$ from NoRH is shown. Lower left: NoRH contour superimposed on the NoRH $34 \mathrm{GHz}$ image, with the rectangular region chosen for calculating $T_{\mathrm{b}}$ (same region as above). Right: Time profile of $T_{\mathrm{b}}$ averaged over the rectangular region. The prominence starts to brighten at approximately 03:50 UT and is increasing during the slow-rise phase of the eruption.

we cannot identify the corresponding non-thermal emission in microwave images at the slow-rise onset. However, in order to detect non-thermal effects in microwave emission, the emitting electrons would need to have very high energies of several hundred $\mathrm{keV}$ to $\mathrm{MeV}$, depending on the magnetic field strength. We argue that the electron acceleration is not strong enough to generate the microwave emission at this stage.

Non-thermal brightenings in the microwave images are observed later, after 04:40 UT, during the fast-rise phase. Figure 10, showing $34 \mathrm{GHz}$ contours overplotted at 04:44 UT on a TRACE image, indicates the site of the non-thermal emission at this time.

\section{Results}

We have observed the initiation and early evolution of an erupting prominence associated with an M-class flare and a strong CME. With good wavelength coverage available for this event, the study encompassed EUV, X-ray as well as microwave emission. Our multi-wavelength analysis of the erupting event on 2005 July 27 has revealed the following:

1. A small X-ray enhancement in GOES and RHESSI lightcurves occurs almost simultaneously with the onset of the slow-rise phase, close to the rising part of the prominence. From RHESSI spectral modeling at the time of the brightening (spatially integrated), we calculated $E M$ to be $10^{46} \mathrm{~cm}^{-3}$ and $T \sim 15 \mathrm{MK}$. The thermal energy of the observed brightening was estimated to be of the order $\sim 10^{29}$ erg. The high-energy tail of RHESSI spectra (above $10 \mathrm{keV}$ ) provides evidence of non-thermal emission during the precursor brightening. The spectra would have not been well fitted by only using a thermal component. It was necessary to include in the model a non-thermal component with a low power-law index of the non-thermal component of $\sim 1.7$.

2. The prominence is observed to brighten in microwave NoRH images at the same time as the pre-eruption X-ray enhancement and, approximately, at the onset of the slow-rise phase. For the region chosen in our analysis, the brightness temperature at $34 \mathrm{GHz}$ was found to increase from $\sim 6500 \mathrm{~K}$ to over $8000 \mathrm{~K}$ during the eruption.

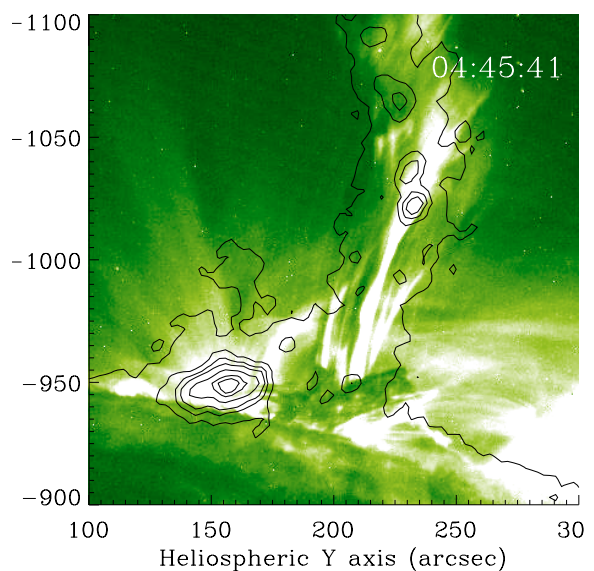

Fig. 10. NoRH $34 \mathrm{GHz}$ contours overplotted on a near-simultaneous TRACE image (which has been corrected for pointing) at 04:45:41 UT showing the site of the non-thermal brightening during the fast-rise phase of the eruption. Both NoRH contours and corrected TRACE image have been rotated $90^{\circ}$ clockwise.

3. An enhancement in EUV is observed in TRACE images, $\sim 30 \mathrm{~min}$ after the slow-rise onset. This brightening is located at the same position as the pre-eruption X-ray brightening (indicating plasma which has cooled down to EUV temperatures). Around this time, an upward shooting magnetic bright thread is also observed at the anchored prominence footpoint, slightly before the fast-rise onset. During the fastrise eruption phase, we observe EUV brightenings rapidly evolving along the accelerating prominence.

4. The impulsive stage of the flare occurs during the fast-rise eruption phase of the prominence. At this time, non-thermal emission is observed spatially coincident in both RHESSI hard X-ray images and NoRH microwave images, with the site of emission source different from the precursor X-ray brightening.

5. During the slow-rise phase, lasting for at approximately $34 \mathrm{~min}$, the rising part of the prominence moves at a constant speed of $4.8 \mathrm{~km} \mathrm{~s}^{-1}$ away from the Sun's centre. During the fast-rise phase, the speed increases linearly, reaching $300 \mathrm{~km} \mathrm{~s}^{-1}$ in less than 10 minutes. The height-time profile of the erupting prominence correlates very well with the time profile of soft X-ray emission detected by GOES.

A chronological description of the event revealed by our multiwavelength analysis is summarised in Table 2.

\section{Discussion and conclusions}

In this paper we have presented the first detailed multiwavelength analysis of the incipient stages of a prominence eruption. The position of the prominence and availability of a wide range of multi-wavelength data enabled a close investigation of the pre-eruption stages as well as early evolution of the event. In our study, we made use of high-resolution and highcadence observations in EUV from TRACE, X-ray spectra and images from RHESSI, as well as NoRH microwave images.

Despite lacking direct information about the magnetic configuration of the eruption site (the filament being situated on the limb), we discuss our results in the context of eruption trigger mechanisms proposed in the literature, based on emission signatures of magnetic processes such as reconnection. Breakout models (Antiochos 1998) predict that reconnection occurs high 
Table 2. Multi-wavelength observations time-line summary of the 2005 July 27 event.

\begin{tabular}{|c|c|c|c|}
\hline Time (UT) & EUV & X-ray & Microwave \\
\hline $03: 50$ & & brightening at $\mathrm{FP}^{*}$ & increase in $T_{\mathrm{b}}{ }^{* *}$ \\
\hline 03:58 & noticeable slow-rise & & \\
\hline 04:28 & brightening at FP & & \\
\hline 04:29 & sudden heating of magnetic thread & & \\
\hline $04: 32$ & onset of fast-rise & & \\
\hline $04: 45$ & fast evolving brightenings & & $\begin{array}{l}\text { non-thermal brightening } \\
\text { (site shifted north of FP) }\end{array}$ \\
\hline 04:50 & & $\begin{array}{c}\text { hard X-ray emission } \\
\text { (site shifted north of FP) }\end{array}$ & \\
\hline 05:01 & & maximum SXR flux (flare peak) & \\
\hline
\end{tabular}

above the prominence before the eruption. Tether-cutting mechanisms (see e.g. Moore \& Roumeliotis 1992) imply that reconnection beneath the prominence unleashes the explosion. Prior to the explosion, the core magnetic field suspending the filament is in force-free equilibrium (Moore \& Sterling 2005), the magnetic pressure being balanced by the field's own magnetic tension as well as tension and pressure from surrounding fields.

The prominence eruption on 2005 July 27 is consistent with the double-phased evolution of previous filaments studied by e.g. Sterling \& Moore (2003, 2004a,b, 2005) and Gretchnev et al. (2006). The first question refers to the onset of the slow-rise phase of the prominence eruption. The small X-ray enhancement seen in GOES and RHESSI light-curves corresponds in time with the observed slow-rise onset. From RHESSI spectral analysis we found evidence at this time of hot plasma with energies above $10 \mathrm{keV}$ and non-thermal particles. We infer that a reconnection event happened at the time of the X-ray brightening, destabilising the prominence which proceeded to a slow-rise phase. The reconnection apparently occurred not in the overlying loop arcades, as would be predicted by breakout model, but beneath its southern footpoint, a scenario which is more similar to the tether-cutting representation.

Previous evidence of both thermal and non-thermal emission in the pre-flare phases of an impulsive GOES class X flare has also been found by Asai et al. (2006), indicating that the early energy release mechanism may be accompanied by particle acceleration. Asai et al. (2006) argued that, although with a milder effect, non-thermal emission present in pre-flare phases may also be present in the early stages of milder flares, such as the flare accompanying the 2005 July 27 event.

The second issue to address is what causes the transition from the slow, steady motion of the prominence to the fast, accelerated motion. We observe a suggestive EUV bright filament strand just before the onset of the fast-rise phase. The magnetic field line associated with the observed upward shooting strand appears as if it were suddenly cut off, while the anchored, slowrising prominence is unleashed and begins to accelerate. Our interpretation is that the bright strands observed in EUV threading the prominence must be heated during the fast-rise phase indicating that these field lines undergo further reconnection. We propose the following scenario for the prominence evolution during the fast-rise phase:

- Reconnection event(s) producing brightenings near the southern prominence footpoint (either at one end or middle of the prominence) trigger the fast-rise, probably by weakening the stabilising tension force.
- As part of the prominence (near the first reconnection event) rises, it stretches the field lines that are connected to the photosphere and have anchored the prominence prior to eruption.

- The stretched field lines undergo further reconnection which proceeds along the prominence (either from one end to the other end, or from middle to both ends), causing not only the heating of the filament threads, but also further destabilisation and acceleration of the prominence.

Moore \& Sterling (2005) describe a similar positive feedback loop process as "runaway reconnection": the prominence rises, reconnection is then induced, which in turn accelerates the prominence rise and so on. In our analysis, this process seems to proceed from the southern part (where the precursor X-ray brightening is observed) to the northern part of the prominence, in a "domino"-like effect. The mild increase in heating determined from the evolution of $T_{\mathrm{b}}$ suggests that, during the slowrise phase, the eruption may also be subject to such type of positive feedback process of a milder form ("slow-runaway reconnection", Moore \& Sterling 2005).

It has been argued before that reconnection and eruption may be coupled dynamically (Ohyama \& Shibata 1997; Zhang et al. 2001; Chen \& Shibata 2000). We suspect that the signature of reconnection observed just before the onset of the fast-rise phase (the EUV upward shooting strand) may be closely related to the eruption having been already triggered (and in the slow-rise phase).

Several 2D (2.5D) eruption scenarios proposed in the literature involve emerging flux (e.g. Chen \& Shibata 2000; Lin et al. 2001). One may criticise these models since emerging flux can never be as long as the filament channel itself (the models being restricted to 2D). From our analysis, we infer that reconnection, which may or may not be associated with an emerging flux, could in fact destabilise the entire prominence (even initially localised only at one footpoint).

We believe that brightenings in EUV (which indicate heating) are common in the pre-eruption stages of filaments (Moore et al. 2001). However, there are insufficient studies performed with high-cadence data to reach a final conclusion. We are also uncertain of how often the observed heating is located beneath the erupting prominence. Sterling et al. (2001) examined the pre-eruption activity of a quiet-region filament, finding the acceleration phase of the filament starting before they observe an EUV brightening under the erupting filament (reconnection was seen as a by-product of the eruption). However, Sterling \& Moore (2004a) assessed the findings of Sterling et al. (2001) as possibly premature, due to the poor cadence provided by EIT. 
Their subsequent analysis of the same event was also inconclusive as to whether reconnection began early enough to cause the fast-rise phase of the eruption.

Moon et al. (2004) have also examined the initial stages of a flare-associated filament eruption in high-cadence and high resolution $\mathrm{UV}, \mathrm{H} \alpha$ images as well as magnetograms, correlating the pre-flare activity with canceling magnetic features (CMFs) in the photospheric field. Moon et al. (2004) concluded that the transient precursor brightenings observed near one footpoint of the filament are consequences of low-atmosphere magnetic reconnection. The analysis of our prominence has revealed evidence of high-energy (above $10 \mathrm{keV}$ ), probable non-thermal photons in the pre-eruption stages of the flares, which would imply that magnetic reconnection occurred higher in the corona in order for this emission to be detected.

We know of only one recent study that has used high-cadence TRACE data to resolve pre-eruption brightenings (Sterling \& Moore 2005). Most previous studies (Sterling \& Moore 2003, 2004a), using EIT data with worse time cadence, might have failed to observe such pre-eruption features.

We found that the height-time profile of the erupting prominence correlates very well with the time profile of soft X-ray emission peak detected by GOES. Zhang et al. (2001) also report a good correlation between the SXR flare lightcurves and the motion of associated CMEs. Kundu et al. (2004), however, have found that the fast-rise phase of an erupting filament ceases 30 minutes before the peak of the X-ray emission. In our analysis, the erupting prominence follows the motional pattern of flare-associated CMEs (Zhang et al. 2001).

Although 2D EUV and microwave images of the prominence may suggest an asymmetric eruption (one southern end rising while the other remains fixed), it is difficult to infer how much the prominence extends behind the limb. Therefore, we do not dismiss the possibility that the observed southernmost part of the prominence represents a barb by which the prominence is initially connected, while its other end (invisible to the observer) is connected behind the limb. $\mathrm{H} \alpha$ images taken by the Kanzelhöhe Solar Observatory ${ }^{2}$ several days after the eruption show a relatively complex structure of the filament channel, most likely residing at the same location of the erupted prominence. Therefore, we are reluctant to classify this eruption as an asymmetric one. Discussing 3D scenarios of filament eruptions in both a symmetric and an asymmetric case, Tripathi et al. (2006) have addressed the issue of what might cause either of these event types. While an emerging bipole detected in magnetograms at the middle of the symmetric filament channel was found to be a likely trigger for this eruption, no conclusive observations were reported in the asymmetric case. Regardless of the type of eruption - symmetric or asymmetric - to the best of our knowledge, the present work provides the first detailed multi-wavelength investigation of the early phases and evolution of a prominence eruption.

From previous studies of eruption triggers (e.g. Sterling \& Moore 2005), as well as from the present analysis, it became clear that high-resolution and high-cadence observations in multi-wavelength emission (chromospheric, transition region and coronal) are required. At present, TRACE images in EUV, complemented by X-ray spectral and imaging RHESSI data as well as microwave NoRH images allow for a detailed analysis. It is expected that future missions such as STEREO, capable of revealing the $3 \mathrm{D}$ configuration of prominences, and SOLAR-B, providing high spatial and temporal resolution images and magnetograms, will further our understanding of filament eruption initiation and its relationship to flare and CME triggers.

Acknowledgements. The authors wish to thank the anonymous referee for a careful reading of our manuscript and the useful suggestions. We acknowledge the use of data from TRACE, SoHO, RHESSI, GOES and NoRH. TRACE and RHESSI are NASA Small Explorer missions. SoHO is an international cooperative project between ESA and NASA. We thank Brian Dennis for help with RHESSI data analysis and Peng-Fei Chen for useful comments and suggestions. $\mathrm{CC}$ is grateful for scholarship support received from the University of Cambridge Overseas Trust, an Isaac Newton Studentship from the Cambridge Institute of Astronomy and an Overseas Research Student Award. DT and HEM acknowledge support from PPARC. HI is funded by a Research Fellowship offered by the Japan Society for the Promotion of Science for Young Scientists.

\section{References}

Antiochos, S. K. 1998, ApJ, 502, L181

Asai, A., Nakajima, H., Shimojo, M., et al. 2006, PASJ, 58, 1, L1

Chen, P. F., \& Shibata, K. 2000, ApJ, 545, 524

Chifor, C., Mason, H. E., Tripathi, D., Isobe, H., \& Asai, A. 2006, in Proc. of ESA SoHO 17, in press

Delaboudinière, J. -P., Artzner, G. E., Brunaud, J., et al. 1995, Sol. Phys., 162, 291

Feynman, J., \& Martin, S. F. 1995, J. Geophys. Res., 100, 3355

Gallagher, P. T., Dennis, B. R., Krucker, S., Schwartz, R. A., \& Tolbert, A. K. 2002, Sol. Phys., 210, 341

Gretchenev, V. V., Uralov, A. M., Zandanov, V. G., Barnov, N. Y., \& Shibasaki, K. 2006, PASJ, 58, 69

Handy, B. N., Acton, L. W., Kankelborg, C. C., et al. 1999, Sol. Phys., 187, 229 Hurford, G. J., Schmahl, E. J., Schwartz, R. A., et al. 2002, Sol. Phys., 210, 61 Illing, R. M. E., \& Hundhausen, A. J. 1985, J. Geophys. Res., 90, 275 Kundu, M. R., White, S. M., Garaimov, V. I., et al. 2004, ApJ, 607, 530 Landi, E., Del Zanna, G., Young, P. R., et al. 2006, ApJS, 162, 261

Lin, J., Forbes, T. G., \& Isenberg, P. A. 2001, J. Geophys. Res., 106, 25053

Lin, R. P., Dennis, B. R., Hurford, G. J., et al. 2002, Sol. Phys., 210, 3

Moon, Y. -J., Chae, J., Choe, G. S., et al. 2004, JKoAS, 37, 41

Moore, R. L. 1998, ApJ, 324, 1132

Moore, R. L., \& Roumeliotis, G. 1992, in Eruptive Solar Flares, ed. Z. Svestka, et al., 399, 69

Moore, R. L., \& Sterling, A. C. 2005, in Solar Eruptions and Energetic Particles, ed. N. Gopalswamy (Washington DC: AGU)

Moore, R. L., Sterling, A. C., Hudson, H. S., \& Lemen, J. R. 2001, ApJ, 552, 833

Nakajima, H., Nishio, M., Enome, S., et al. 1994, in Proc. IEEE, 82, 705

Ohyama, M., \& Shibata, K. 1997, PASJ, 49, 249

Phillips, K. J. H., Chifor, C., \& Landi, E. 2005, ApJ, 626, 110

Phillips, K. J. H., Chifor, C., \& Dennis, B. R. 2006, ApJ, 647, 1870

Priest, E. R., \& Forbes, T. G. 2002, A\&ARv, 10, 313

Rao, A. P., \& Kundu, M. R. 1977, Sol. Phys., 55, 161

Rust, D. M. 1976, Sol. Phys., 47, 21

Shibata, K. 1999, Ap\&SS, 264, 129

Sterling, A. C., \& Moore, R. L. 2003, ApJ, 599, 1418

Sterling, A. C., \& Moore, R. L. 2004a, ApJ, 602, 1024

Sterling, A. C., \& Moore, R. L. 2004b, ApJ, 613, 1221

Sterling, A. C., \& Moore, R. L. 2005, ApJ, 630, 1148

Sterling, A. C., Moore, R. L., \& Thompson, B. J. 2001, ApJ, 561, L219

Svestka, Z. 2001, Space Sci. Rev., 95, 135

Tripathi, D. 2005, Ph.D. Thesis University of Göttimgen, Copernicus GMBH

Tripathi, D., Isobe, H., \& Mason, H. E. 2006, A\&A, 453, 1111

Wang, Y.-M., \& Sheeley, N. R., Jr. 1999, ApJ, 510, 157

Zhang, J., Dere, K. P., Howard, R. A., Kundu, M. R., \& White, S. M. 2001, ApJ, 559,452 